EDITORIAL

\title{
El difícil mercado de una necesaria publicación científica
}

\section{Leonardo Romero, Editor Jefe}

\section{Un escenario adverso}

Las revistas científicas sensu stricto publican esencialmente los denominados artículos primarios o memorias científicas originales $^{1}$. Estos artículos son escuetos, minuciosos, con lenguajes técnicos especializados; son comunicaciones sobre observaciones o descubrimientos provenientes de investigaciones y que por lo general son solamente valorados por otros investigadores de la misma especialidad. Estas características hacen de las revistas científicas un objeto de difícil circulación, muchas de ellas solamente se distribuyen a bibliotecas de universidades e institutos especializados. Comparadas con otros medios de comunicación, las revistas científicas tienen muy poco impacto sobre la sociedad, y esto es una constante preocupación para los editores y una amenaza a la existencia de las revistas científicas, ya que con un mercado reducido sobreviene el incremento de los costos y su posible extinción. Superar esta situación es un reto, aunque algunas revistas científicas, principalmente del área medica, han elaborado estrategias que les permiten acercarse al publico en general, publicando artículos de interés general, relacionados al avance de las investigaciones, nuevos medicamentos, enfermedades emergentes, etc., logrando sobretodo subrayar su rol e importancia dentro de sociedad. Para las revistas de ciencias biológicas también se ha sugerido un enfoque similar².

Por otro lado, la cantidad de investigadores, las investigaciones, el número de artículos y las revistas científicas, guardan una clara relación. El estudio Worldwide Scientific Publishing Activity ${ }^{3}$, en el que se analiza la proveniencia más de 4 millones de artículos publicados en la base de datos MEDLINE desde 1986 al 2001, califica al Perú entre aquellos con menor actividad científica (100 publicaciones por cada millón de habitantes).
La producción científica nacional también puede analizarse por el número de revistas científicas nacionales. Esto se puede observar en el registro de la base de datos Latindex ${ }^{4}$. El Perú, en el Directorio Latindex reporta un total de 99 revistas científicas vigentes, de las cuales 34 están también publicadas on line, 10 revistas están dedicadas a ciencias biológicas y solamente dos, la Revista Peruana de Entomología y la Revista Peruana de Biología cumplen con las normas para figurar en el Catálogo Latindex (selección y clasificación según criterios internacionales de calidad editorial), además de ser las únicas indizadas en las bases de datos BIOSIS.

\section{Una necesidad dentro de un nuevo orden}

La publicación de un artículo en una revista científica puede considerarse como la transmisión y acreditación de los resultados de las investigaciones y a la vez parte integrante de la generación de conocimientos. Es por este motivo que necesitamos producir más información, ponerla a disposición de la sociedad y explicar la importancia de la actividad científica en el desarrollo del país. En las ultimas décadas la humanidad ha sido envuelta por la vorágine de tecnologías que han cambiado muchos conceptos de aprendizaje e investigación, ya no es propiedad de elites sino de toda la sociedad, la información se adquiere y circula a velocidades no imaginadas antes. Las revistas científicas, como medio de información, también han desarrollado cambios, algunos no tan positivos, como el incremento en costo y la brecha de información existente entre los países desarrollados y los no desarrollados. El año pasado la UNESCO propuso oficialmente en un documento el paradigma de las «sociedades del conocimiento» ${ }^{5}$, con la idea central de interpretar un nuevo escenario social que comprenda nuevas dimensiones sociales, éticas 
y políticas mucho mas vastas y que permitirán el desarrollo de la Investigación - Innovación y Desarrollo (IID), en este documento se resalta la importancia de la investigación básica, la documentación y la diseminación en las revistas científicas, como uno de los elementos necesarios para cumplir con el logro de la idealizada sociedad del conocimiento.

\section{Un articulo científico estratégico}

Desde 1998 la Revista Peruana de Biología ha publicado 216 artículos, principalmente sobre biodiversidad. De ellos el $80 \%$ son de carácter descriptivo, el 34\% de taxonomía, incluyendo 9 artículos con descripciones de especies nuevas. Los trabajos dedicados al estudio de animales son el $45 \%$ (incluyendo parásitos), mientras los artículos dedicados a plantas y los microbiológicos son el 16 y $11 \%$ respectivamente. Aunque aún son pocos trabajos, estos representan en su mayoría una contribución al conocimiento de la biodiversidad, un recurso natural que ha cobrado especial interés por el uso que puede dársele con la biotecnología y por lo tanto participar activamente en el desarrollo económico del país. El conocimiento de la biodiversidad no solamente puede ser aprovechado por la biotecnología, sino que nos ayuda a evitar catástrofes o crisis ecológicas o sanitarias, prueba de ello es el interés por las denominadas especies allien o invasoras, dentro de las que podemos encontrar animales, plantas y organismos causante de enfermedades. Hay que resaltar que considerar una estrategia de desarrollo científico en el campo de la biodiversidad y biotecnología no es una proposición decorativa para un país Megadiverso como el nuestro. Por el contrario actualmente países como Brasil han incrementado las facilidades y medios para la formación de taxónomos y sistemáticos, logrando tener mas investigadores en esas áreas que en otros países desarrollados 6 . Justamente por que los taxónomos y sistemáticos son considerados parte de una estrategia de Investigación-Desarrollo en el conocimiento, aprovechamiento y conservación de la biodiversidad.

\section{Aún así un difícil producto}

Los artículos referidos a taxonomía y descripción de nuevas especies son de carácter especial y algunos investigadores pueden considerarlos como documentos legales ${ }^{7}$, debido a que se guían por códigos y normas estrictas y por la trascendencia que tienen en el campo científico. La descripción de una nueva especie tiene un gran impacto sobre otros artículos que analicen la biodiversidad de un área geográfica, su conservación, la determinación de especies amenazadas, endemismos, filogenia, entre otros. Estos artículos son los cimientos de bases de datos como el antbase. org $^{8} \mathrm{y}$ otras propuestas como el ZooBank ${ }^{9}$; ambos esfuerzos ligados al Global Biodiversity Information Facility y que promueven tecnologías de información para compartir datos con la finalidad de conservar la biodiversidad. A pesar de la conspicua importancia de la nominación de una nueva especie y de los estudios taxonómicos, la revista donde éstos se escriben sigue siendo de escasa circulación, aunque sean documentos casi imperecederos.

1 UNESCO. Guía para la redacción de artículos científicos destinados a la publicación. 2 ed. París: UNESCO, 1983 (PGI-3/WS/10)

${ }^{2}$ Watson D. M. 2004. Beyond impact factor. Frontiers in Ecology and the Environment: 2(7): 346-347.

${ }_{3}^{3}$ Perez-Iratxeta C. \& M. A. Andrade. 2002. Worldwide Scientific Publishing Activity. SCIENCE 297: 519

${ }^{4}$ http://www.latindex.unam.mx

5 UNESCO. 2005. Hacia las sociedades del conocimiento. http//www.unesco.org/publications

${ }^{6}$ De Carvalho M. R. et al. 2005 Revisiting the Taxonomic Impediment. SCIENCE 307: 353

${ }^{7}$ Minelli A. 2005. Publications in taxomony as scientific papers and legal documents. Proceedings of the California Academy of Science. 56 (20)suppl.: 225-231

${ }^{8}$ Agosti, D., \& N. F. Johnson. (Editors). 2005. Antbase. World Wide Web electronic publication. antbase.org, version (05/2005).

9 Polaszek A. et al. 2005. ZooBank, the open-access register for zoological taxonomy: Technical Discussion Paper. Bulletin of Zoological Nomenclature 62(4): 210-220 